\title{
Overall Distribution of Trauma-Related Deaths in Berlin 2010: The Weakest Links of the Chain of Survival are Emergency Medicine and Critical Care: Reply
}

\author{
Christian Kleber • Moritz Giesecke • \\ Claas Buschmann
}

Published online: 18 October 2012

(C) Société Internationale de Chirurgie 2012

\section{Dear Editor,}

We are very happy to have received such a great response since the publication of our article about traumatic deaths in Berlin 2010 [1]. Furthermore, we expect more controversy and scientific discussion after publication of the second part, preventable trauma deaths (article in review, World Journal of Surgery).

In our opinion, the major finding of our first study is the underestimation of the importance of preclinical trauma management. The results of our second study will clarify that the quality of preclinical trauma management is overestimated, and therefore the significance and potential to reduce traumatic deaths is not recognized. With the primary focus on advancements in critical care, one of the two "hot spots" of trauma management, the main problem, preclinical trauma management, is disregarded [2]. In a recent study, we revealed the need for individualized preclinical treatment of trauma patients with invasive preclinical measures, e.g., intubation and chest tubing [3]. Therefore, we conclude that the education of paramedics and emergency physicians, and the active contribution of trauma surgeons to preclinical trauma management are crucial factors for improving the quality of national trauma management, and that adding these components has the potential to significantly lower mortality after severe trauma. All organizational, structural, and management efforts (national trauma network, trauma registry, national interdisciplinary guideline)

C. Kleber $(\bowtie) \cdot$ M. Giesecke

Center for Musculoskeletal Surgery, Campus Virchow

Klinikum, Charité-Universitätsmedizin, Augustenburger Platz 1,

13353 Berlin, Germany

e-mail: christian.kleber@charite.de

C. Buschmann

Institute of Legal Medicine and Forensic Sciences,

Charité-Universitätsmedizin, Berlin, Germany rely on effective preclinical trauma management. Thus, we have to secure the preclinical treatment of trauma patients and advocate for their needs.

Referring to the letter from Brambillasca et al. [4] we totally agree with their striving for structures and national protocols necessary for a modern trauma system, but the basis of all is an intact chain of survival. The weakest link is always crucial, and in our study, preclinical trauma care was the weakest link. Therefore, we recommend advancing preclinical trauma management further by educating paramedics and emergency physicians in the administration of safe and effective preclinical treatment of patients after severe trauma. Self-evidently there are regional or national differences demanding an analysis of local trauma systems to reveal their weakest link(s). To our minds, the new bimodal temporal distribution of traumatic deaths is not only a regional phenomenon but also the logical consequence of continuous advancement in the treatment and logistics of trauma patients revealing the two hot spots, or weakest links: preclinical trauma management and intensive care medicine.

\section{References}

1. Kleber C, Giesecke MT, Tsokos M et al (2012) Overall distribution of trauma-related deaths in Berlin 2010: advancement or stagnation of German trauma management? World J Surg 36:21252130. doi: 10.1007/s00268-012-1650-9

2. Kleber C, Schaser KD, Haas NP (2011) Surgical intensive care unit-the trauma surgery perspective. Langenbecks Arch Surg 396: 429-446

3. Kleber C, Lefering R, Kleber AJ et al (2012) Rescue time and survival of severely injured patients in Germany. Unfallchirurg. doi: $10.1007 / \mathrm{s} 00113-011-2132-5$

4. Brambillasca P Coccolini F, Catena F et al (2012) Trauma care: not all countries are equal. World J Surg. doi:10.1007/s00268- 